\title{
A simple 5-DoF MR-compatible motion signal measurement system
}

\author{
Soon-Cheol Chung • Hyung-Sik Kim • Jae-Woong Yang • Su-Jeong Lee • \\ Mi-Hyun Choi • Ji-Hye Kim • Hong-Won Yeon • Jang-Yeon Park • Jeong-Han Yi • \\ Gye-Rae Tack
}

Published online: 13 April 2011

(C) Psychonomic Society, Inc. 2011

\begin{abstract}
The purpose of this study was to develop a simple motion measurement system with magnetic resonance (MR) compatibility and safety. The motion measurement system proposed here can measure 5-DoF motion signals without deteriorating the MR images, and it has no effect on the intense and homogeneous main magnetic field, the temporalgradient magnetic field (which varies rapidly with time), the transceiver radio frequency (RF) coil, and the RF pulse during MR data acquisition. A three-axis accelerometer and a twoaxis gyroscope were used to measure 5-DoF motion signals, and Velcro was used to attach a sensor module to a finger or wrist. To minimize the interference between the MR imaging system and the motion measurement system, nonmagnetic materials were used for all electric circuit components in an MR shield room. To remove the effect of RF pulse, an amplifier, modulation circuit, and power supply were located in a shielded case, which was made of copper and aluminum. The motion signal was modulated to an optic signal using pulse width modulation, and the modulated optic signal was transmitted outside the MR shield room using a high-intensity light-emitting diode and an optic cable. The motion signal was recorded on a PC by demodulating the transmitted optic signal into an electric signal. Various kinematic variables, such as angle, acceleration, velocity, and jerk, can be measured or calculated by using the motion measurement system devel-
\end{abstract}

S.-C. Chung $\cdot$ H.-S. Kim $\cdot$ J.-W. Yang $\cdot$ S.-J. Lee $\cdot$ M.-H. Choi J.-H. Kim $\cdot$ H.-W. Yeon $\cdot$ J.-Y. Park $\cdot$ J.-H. Yi $\cdot$ G.-R. Tack $(\bowtie)$ Department of Biomedical Engineering, Research Institute of Biomedical Engineering, College of Biomedical and Health Science, Konkuk University,

322 Danwol-dong,

Chungju, Chungbuk 380-701, Korea

e-mail: grtack@kku.ac.kr oped here. This system also enables motion tracking by extracting the position information from the motion signals. It was verified that MR images and motion signals could reliably be measured simultaneously.

Keywords MR compatible $\cdot 5$-DoF motion signal · Three-axis accelerometer - Two-axis gyroscope

\section{Introduction}

To describe, analyze, and assess human movements, many studies have been conducted from biomechanical, motor control, and neurological points of view (Abler, Erk, \& Walter, 2007; Jones \& Rose, 2004; Rocca et al., 2007; Vangheluwe, Suy, Wenderoth, \& Swinnen, 2005). These days, most neurological studies use functional magnetic resonance imaging (fMRI). In early neurological studies, motion signals were rarely obtained during fMRI experiments, due to the restriction of the strong magnetic field environment. Only a few studies on brain function during movement, which were based on simple tasks such as changing finger and wrist speed or involved imaginary finger sequencing, have been conducted (Cramer et al., 2002; Debraere, Wenderoth, Sunaert, Van Hecke, \& Swinnen, 2004; Rocca, 2007; Van Duinen, Remco, Natasha, \& Inge, 2007). Since motion data were not obtained in these studies, an accurate analysis of the relationship between human movement and brain function has not been accomplished so far.

The development of a simple system for motion data acquisition in a magnetic resonance (MR) environment is in progress, with the goal of investigating the relationship between movement and brain function. A grip force 
measurement system was developed using a force transducer based on hydraulic pressure (Liu et al., 2000). Other researchers have used an accelerometer (Morgen et al., 2004) and a potentiometer (Carey et al., 2002) to measure single-finger motion along a single axis in conjunction with acquiring MR data. These systems could not measure kinematic variables directly and were limited by the measurement accuracy of repeated simple movements. Also, in another study the single-axis angular velocity of five fingers was measured and jerk information extracted (Schaechter, Stokes, Connell, Perdue, \& Bonmassar, 2006). However, the measurement accuracy worsened over time, and the difference in brain activation based on jerk was not identified, since the angular velocity and jerk were measured using only a yaw rate sensor. Although the structure of these measurement systems was simple, the types of measurable kinematic variables were limited, and their results were not accurate.

Recently, using an MR-compatible robot with a haptic interface function, a study was conducted on the neurological mechanisms of active and passive motions (Gassert, Burdet, \& Chinzei, 2008a). Taking advantage of the benefits of robots, such as the available accuracy, precision, and repeatability, studies on motion tracking (Gassert, Burdet, \& Chinzei, 2008b) and motor control and rehabilitation (Yu, Hollnagel, Blickenstorfer, Kollias, \& Riener, 2008) were also carried out. However, the MR-compatible robot has some limitations, due to the presence of numerous scanner types, models, and field strengths. Manufacturers provide an additional difficulty when designing a system for use inside MRI scanners. Since technical solutions for MR-compatible mechatronics and systems are not only complex, large in size, and expensive, but also have small degrees of freedom (DoFs), only a limited number of groups have used this kind of system (Elhawary et al., 2008).

Therefore, it is necessary to develop an MR-compatible motion measurement system that can measure various types of kinematic variables, is appropriate for general purposes, and has a simple structure. In this article, a 5-DoF MRcompatible motion measurement system was proposed that produced minimal MR interference effects, satisfying the desirable characteristics mentioned above - that is, having a simple structure, enabling easy measurement, and extracting information on many kinematic variables, such as angle, velocity, acceleration, and jerk. This system is also expected to offer the capability of motion tracking, using the position data that can be extracted from motion signals.

\section{Development of the 5-DoF MR-compatible motion signal measurement system}

Nonmagnetic materials were used for all electric components in MR shield room in order to satisfy MR

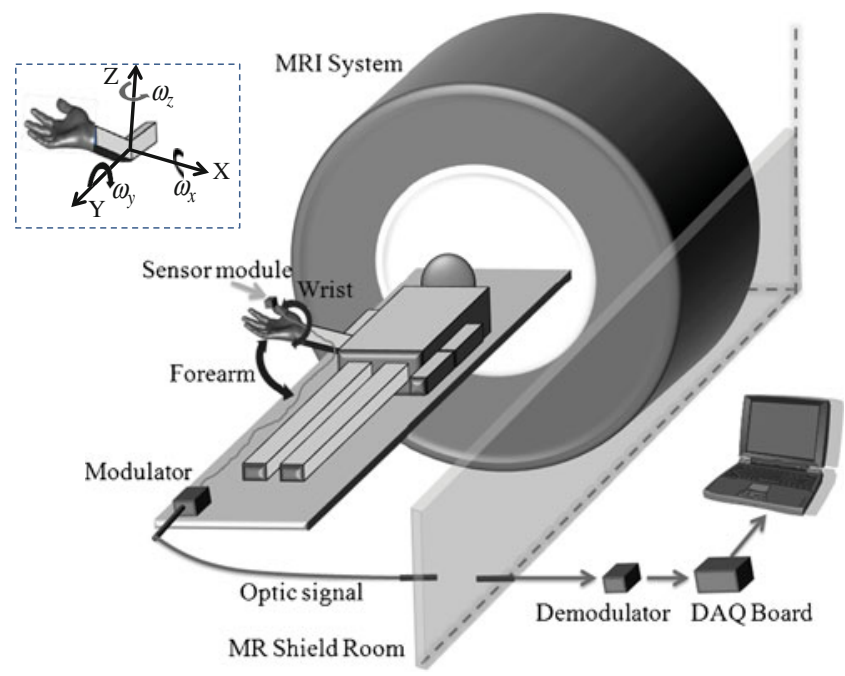

Fig. 1 Configuration of the MR-compatible motion signal measurement system $\left(\omega_{x}, \omega_{y}\right.$ and $\omega_{z}$; i.e., angular velocity in the $x, y$, and $z$ directions, respectively)

compatibility-that is, to minimize interference effects between the motion measurement system and the strong main magnetic field, the temporal gradient magnetic field, and the transceiver radio frequency (RF) coil in MR system. In terms of MR safety, a shielding box made of folding copper (outside) and aluminum (inside) was used to hold an amplifier, modulation circuit, and power supply, because heat might be produced by a current induced by the RF pulse, and it might be harmful for human subjects. The motion measurement system consisted of a sensor module with a three-axis accelerometer (MMA7260Q, Freescale Semiconductor) and a two-axis gyroscope (IDG-650, InvenSense, Inc.), a modulator that amplifies the motion signal and converts it to an optic signal, and a demodulator that converts an optic signal to an electric signal (Fig. 1).

Velcro was used to attach the sensor module to a finger or wrist. Since the sensor module was small (i.e., $10 \times 18.5 \times$ $\left.7.5 \mathrm{~mm}^{3}[\mathrm{~W} \times \mathrm{L} \times \mathrm{H}]\right)$ and its weight with Velcro was only $3.7 \mathrm{~g}$, no complaints about holding it during the experiments were reported from the subjects. To extract acceleration and angle information, a complementary filter algorithm (Hong, 2003) was used, as shown in Fig. 2. Acceleration was measured from the three-axis accelerometer. The measured acceleration signal consisted of alternating current (AC) and direct current (DC) components, which represented pure

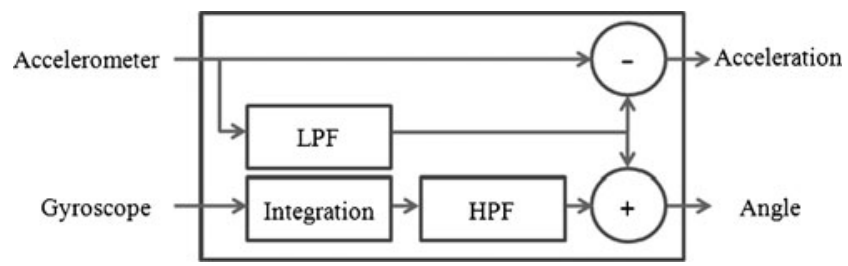

Fig. 2 Block diagram of the complementary filter 
Fig. 3 Block diagram of the modulation and demodulation components

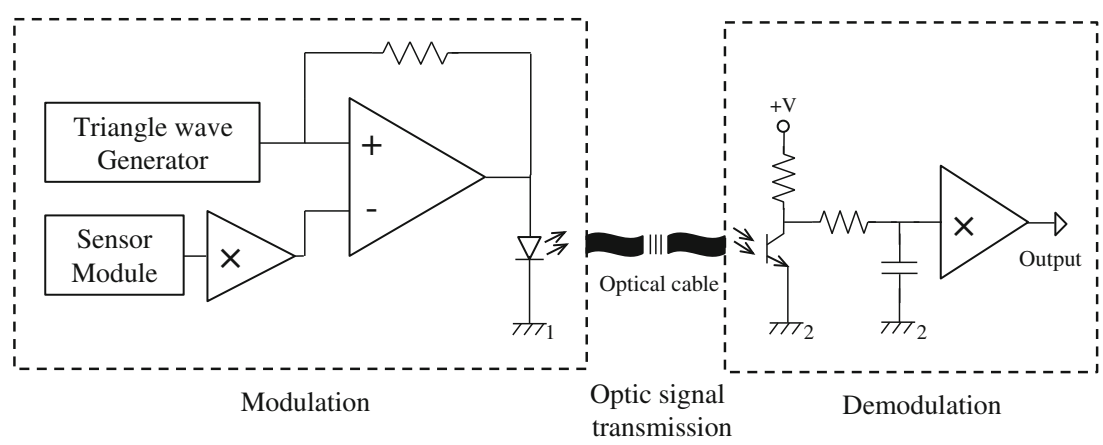

acceleration and gravitational acceleration, respectively. The pure acceleration was determined by subtracting the DC component from the measured signal, where the DC component was obtained by using a $0.4-\mathrm{Hz}$ 5th-order Butterworth infinite impulse response low-pass filter (Baerveldt \& Klang, 1997). Velocity and jerk were also calculated from the acceleration signal. The DC component of the acceleration signal was used as a complementary signal for angle information. First, angle was calculated by using a numerical integration approachthat is, the Runge-Kutta method - based on the angular velocity signals measured from the two-axis gyroscope. After high-pass filtering to remove errors due to drift, which is the low-frequency component from the sensor, the angle signal was finally extracted by comparing the complementary signal (Hong, 2003). Since the degree of variation of drift depends on the environment and temperature, before each experiment the cutoff frequency of the high-pass filter was determined after calibration.

An electric wire was connected between the sensor module and the modulator (Fig. 1). When using a short electric wire, the modulator was placed near the center of the MR magnet, thus possibly inducing noise in both the motion signals and MR images. In the case of using a long electric wire, the noise in the motion signals might have been due to the induced electric field from the electric wire. Therefore, the modulator was placed at the edge of the MR system in order to stably measure motion signals without creating noise in the MR images. Generation of an electric field was prevented by twisting the electric wires between the sensor module and the modulator, and the electric wires were affixed to minimize noise due to vibration during MR image acquisition.
The modulator included the amplifier circuit as well as the circuit that modulates the motion signal to an optical signal. The amplifier circuit consisted only of analog elements and was designed with 10 variable amplifier rates to achieve an optimal gain. A triangular wave with a frequency $(10 \mathrm{KHz})$ higher than the frequency range of the motion signal was put into the comparator, accompanied with the motion signal, and the motion signal was modulated by the pulse width modulation method, which changes the pulse width of a rectangular wave generated from the comparator as an output. This modulated motion signal was converted into an optic signal using a high-intensity light emitting diode and was transmitted outside the MR shield room through an optic cable (Fig. 3). The electric power supply was placed at least $3 \mathrm{~m}$ away from the modulator.

The demodulator received the transmitted optic signal using a photo transistor and converted it to an electric signal using a low-pass filter (Fig. 3). The restored signal was transmitted to a PC by an analog-to-digital converter using a DAQ board (DAQPad-6015, National Instruments). The motion signal was displayed and stored, and LABVIEW (Version 7.1) and MATLAB (Version 7.3) were used for further analysis.

\section{Testing the 5-DoF MR-compatible motion signal measurement system}

Interactive effects in a human subject were tested by measuring MR images and motion signals simultaneously. Images were obtained using the field echo echoplanar imaging (FEEPI) method $(\mathrm{TR} / \mathrm{TE}=3,000 / 35 \mathrm{~ms}$, field of view = $220 \mathrm{~mm}$, matrix $=256 \times 256$, slice thickness $=4 \mathrm{~mm}$, number
Fig. 4 MR images captured during the fast speed movement (Slice A, $16 \mathrm{~mm}$ above Slice B; Slice B, middle axial image; Slice C, $16 \mathrm{~mm}$ below Slice B)
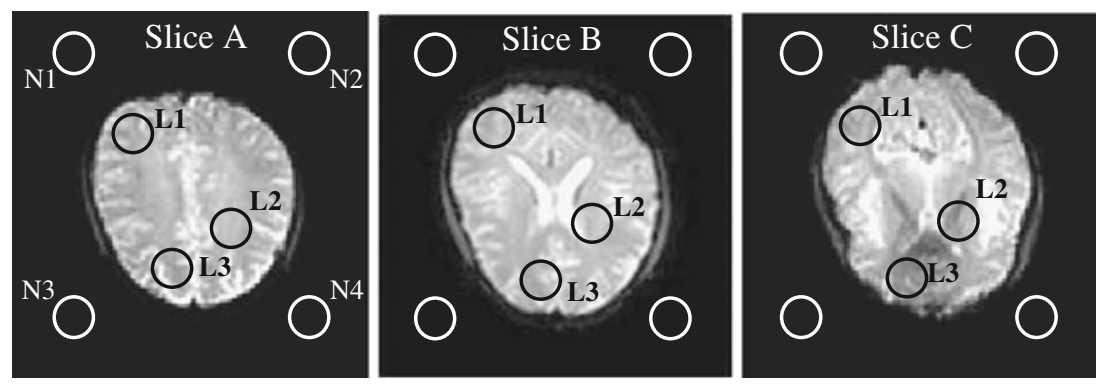
Table 1 Signal-to-noise ratio of MR image for three cases (i.e., without a sensor module, with a sensor module, and with a sensor module + motion) of three slices (A, B, and C) at three regions (L1, L2, and L3)

\begin{tabular}{|c|c|c|c|c|c|c|c|c|c|c|c|c|c|}
\hline & \multicolumn{4}{|c|}{ Slice A } & \multicolumn{4}{|c|}{ Slice B } & \multicolumn{4}{|c|}{ Slice $\mathrm{C}$} & \multirow[t]{2}{*}{ Total Avg. } \\
\hline & L1 & $\mathrm{L} 2$ & L3 & Avg. & L1 & $\mathrm{L} 2$ & L3 & Avg. & L1 & $\mathrm{L} 2$ & L3 & Avg. & \\
\hline Without a sensor module & 174.5 & 198.0 & 194.5 & 189.0 & 193.2 & 189.1 & 183.4 & 188.5 & 201.0 & 172.7 & 185.6 & 186.4 & 188.0 \\
\hline With a sensor module & 173.2 & 197.6 & 192.4 & 187.7 & 193.4 & 188.5 & 181.2 & 187.7 & 201.2 & 171.5 & 183.7 & 185.4 & 186.9 \\
\hline With a sensor module + motion & 171.0 & 196.6 & 191.8 & 186.4 & 191.3 & 187.7 & 179.8 & 186.2 & 199.4 & 172.2 & 182.3 & 184.6 & 185.7 \\
\hline
\end{tabular}

of slices $=30$, flip angle $=90^{\circ}$ ) using a 3.0-T MR system (Achieva 3.0 T TX Series, Philips). MR images were obtained for three cases - that is, without a sensor module, with a sensor module, and with a sensor module while executing motion (i.e., sensor module + motion).

For the execution of motion, extension and flexion of the arm in the $y-z$ plane was performed at three different speedsthat is, slow speed (average 16 times/min), preferred speed (average 32 times $/ \mathrm{min}$ ), and fast speed (average 64 times/min). While motion was executed at each speed for $30 \mathrm{~s}$, measurement of the motion signal was performed simultaneously with the acquisition of MR images at the rate of 5,000 samples/s, using the three-axis accelerometer (Ax, Ay, and Az) and the two-axis gyroscope (Gx and Gy).

Figure 4 shows MR images acquired during the fast speed movement. Slice B is the middle axial image, and Slices A and $\mathrm{C}$ are the axial images $16 \mathrm{~mm}$ above and below Slice B, respectively. In all cases, no deterioration appeared in terms of image quality. The signal-to-noise ratio (SNR) of the MR images was calculated in order to see the effects of the modulator and the movement of the sensor module. As is shown in Fig. 4, the SNR was calculated for three cases (Table 1) - that is, without a sensor module, with a sensor module, and with a sensor module + motion-by dividing each intensity of three regions - that is, Location 1 (L1), Location 2 (L2), and Location 3 (L3) - by the average intensity of four background regions (N1, N2, N3, and N4). Although there was little difference in the SNR between the cells, SNR was slightly reduced in the following order: without a sensor module, with a sensor module, and with a sensor module + motion. Considering that this order is the same as that of the experiment, this slight SNR reduction might be due to the drift of the MR system over time.

Figure 5 shows the motion signal acquired simultaneously with the MR images. In other words, the $y$-axis component of the angle and the $z$-axis component of the acceleration are shown in Fig. 5, which is important information in the flexion and extension of arm. The root mean squared jerk of the $z$-axis component of the acceleration signal was also calculated and plotted. As is shown in Fig. 5 for three different speeds, it was possible to measure and calculate reliable and differentiable motion signals. Due to the

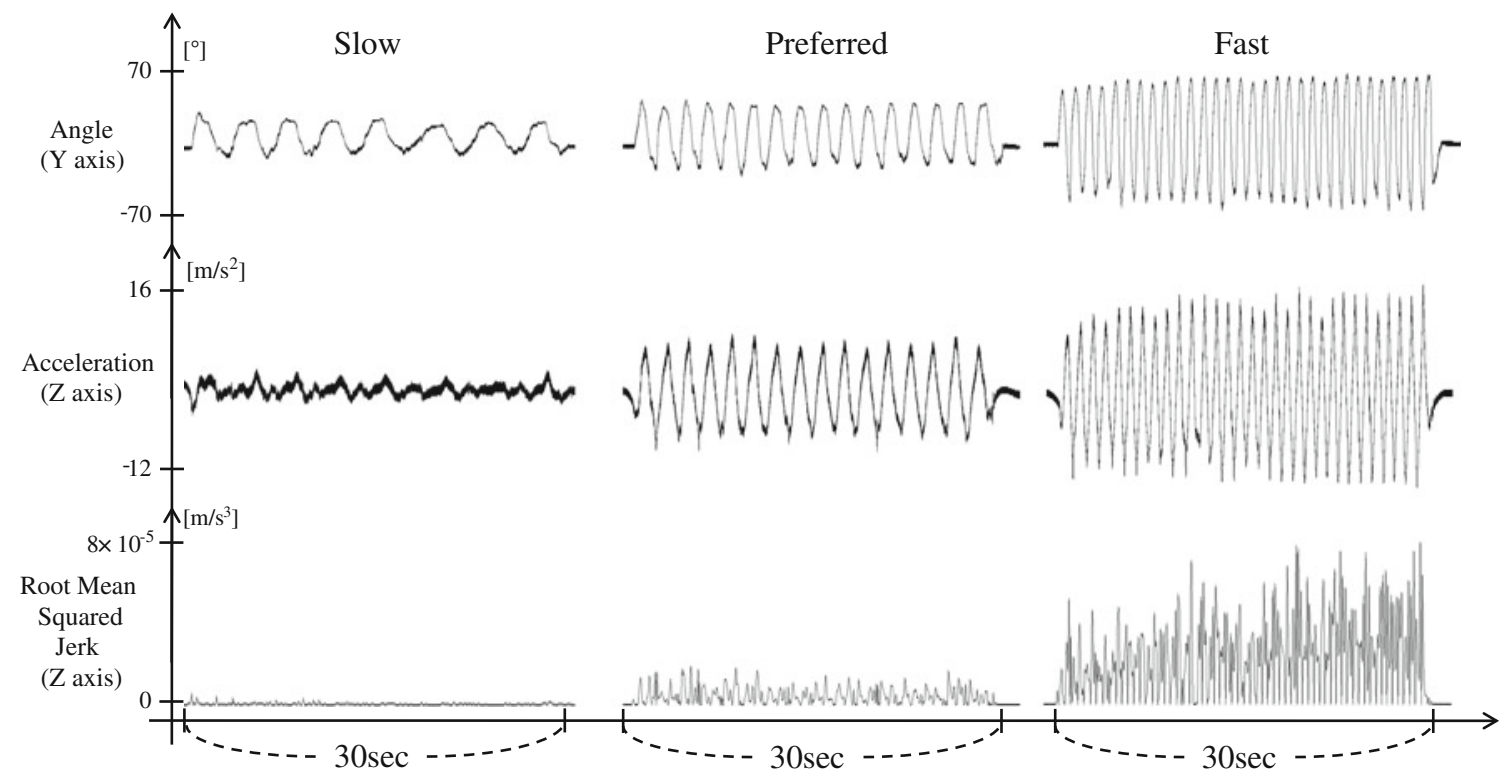

Fig. 5 Motion signal results during extension and flexion of the arm in the $y-z$ plane for the three kinds of speed. Note that different portions of the $y$-axis in the figure show the values for angle ( $y$-axis, degrees), acceleration $\left(z\right.$-axis, $\left.\mathrm{m} / \mathrm{s}^{2}\right)$, and root mean squared jerk $\left(z\right.$-axis, $\left.\mathrm{m} / \mathrm{s}^{3}\right)$ 
constant temperature and humidity in the MR shield room, no drift of the five types of motion signals was observed, even during long use of the system over more than an hour.

\section{Discussion}

In this study, a 5-DoF MR-compatible motion signal measurement system was developed that can extract a variety of kinematic variables, such as angle, acceleration, velocity, and jerk, with simplicity and accuracy using a three-axis accelerometer and a two-axis gyroscope, while minimizing its interference with MR images. Motion tracking would be also available, because position information can be extracted using this system. The MR-compatible and MR-safe properties of the system were achieved by controlling an electric wire as well as by using nonmagnetic materials, analog elements, and a shielding box.

Since the sensor module of the system was small and lightweight, it was possible to measure motion signals at the finger, wrist, and leg using Velcro to secure the motion sensor module. The entire system can be made with low cost, and its direct implementation is available in a clinical MR scanner without any additional installation. Since 25 channels were used in this system, it was possible to simultaneously measure 5-DoF motion signals obtained from each of the five fingers. By adding more channels to the DAQ board, it would also be possible to increase the number of simultaneously measurable motion signals. Therefore, the motion signal measurement system proposed here has several advantages, such as simplicity, generality, and expandability, when compared to a MR-compatible robot. Since inertial sensors - an accelerometer and a gyroscope - were used, this system's accuracy might be somewhat decreased as compared to a robot. However, this limitation can be overcome by using diverse signal processing algorithms.

Although motion tracking using this system was not presented here, it is expected that motion tracking could be easily obtained from the position data, which can be extracted by putting together all of the information about the three acceleration directions, two angles, and arm length. Since the 5-DoF MR-compatible motion measurement system proposed here can reliably measure motion signals using a three-axis accelerometer and a two-axis gyroscope during the acquisition of MR images, it is also expected that this system could be used for the study of the relationship between various kinematic variables and brain function.

Author Note This work was supported by a Mid-career Researcher Program Grant (2009-0084784) through the National Research Foundation of Korea (NRF), funded by the Ministry of Education, Science and Technology (MEST).

\section{References}

Abler, B., Erk, S., \& Walter, H. (2007). Human reward system activation is modulated by a single dose of olanzapine in healthy subjects in an event-related, double-blind, placebo-controlled fMRI study. Psychopharmacology, 191, 823-833.

Baerveldt, A. J., \& Klang, R. (1997). A low-cost and low-weight attitude estimation system for an autonomous helicopter. In Proceedings of the IEEE International Conference on Intelligent Engineering Systems (pp. 391-395). Piscataway, NJ: IEEE Press

Carey, J. R., Kimberly, T. J., Lewis, S. M., Auerbach, E. J., Dorsey, L., Rundquist, P., et al. (2002). Analysis of fMRI and finger tracking training in subjects with chronic stroke. Brain, 125, 773-788.

Cramer, S. C., Weisskoff, R. M., Schaechter, J. D., Nelles, G., Foley, M., Finklestein, S. P., et al. (2002). Motor cortex activation is related to force of squeezing. Human Brain Mapping, 16, 197205.

Debraere, F., Wenderoth, N., Sunaert, S., Van Hecke, P., \& Swinnen, S. P. (2004). Cerebellar and premotor function in bimanual coordination: Parametric neural responses to spatiotemporal complexity and cycling frequency. NeuroImage, 21, 1416-1427.

Elhawary, H., Tse, Z. T. H., Hamed, A., Rea, M., Davies, B. L., \& Lamperth, M. U. (2008). The case for MR-compatible robotics: A review of the state of the art. International Journal of Medical Robotics and Computer Assisted Surgery, 4, 105-113.

Gassert, R., Burdet, E., \& Chinzei, K. (2008a). MRI-compatible robotics. IEEE Engineering in Medicine and Biology Magazine, $27,12-14$.

Gassert, R., Burdet, E., \& Chinzei, K. (2008b). Opportunities and challenges in MR-compatible robotics. IEEE Engineering in Medicine and Biology Magazine, 27, 15-22.

Hong, S. H. (2003). Fuzzy logic based closed-loop strap down attitude system for unnamed aerial vehicle. Sensors and Actuators A, 107, 109-118.

Jones, C. J., \& Rose, D. J. (Eds.). (2004). Physical activity instruction of older adults. Champaign: Human Kinetics.

Liu, J. Z., Dai, T. H., Elster, T. H., Sahgal, V., Brown, R. W., \& Yue, G. H. (2000). Simultaneous measurement of human joint force, surface electromyograms, and functional MRI-measured brain activation. Journal of Neuroscience Methods, 101, 49-57.

Morgen, K., Kadom, N., Sawaki, L., Tessitore, A., Ohayon, J., Frank, J., et al. (2004). Kinematics specificity of cortical reorganization associated with motor training. NeuroImage, 21, 1182-1187.

Rocca, M. A. (2007). Influence of body segment position during inphase and antiphase hand and foot movement: A kinematic and functional MRI study. Human Brain Mapping, 28, 218-227.

Rocca, M. A., Gatti, R., Agosta, F., Tortorella, P., Riboldi, E., Broglia, P., et al. (2007). Influence of body segment position during inphase and antiphase hand and foot movements: A kinematic and functional MRI study. Human Brain Mapping, 28, 218-227.

Schaechter, J. D., Stokes, C., Connell, B. D., Perdue, K., \& Bonmassar, G. (2006). Finger motion sensors for fMRI motor studies. NeuroImage, 31, 1549-1559.

Vangheluwe, S., Suy, E., Wenderoth, N., \& Swinnen, S. P. (2005). Learning and transfer of bimanual multifrequency patterns: Effector-independent and effector-specific levels of movement representation. Experimental Brain Research, 170, 543-554.

Van Duinen, H., Remco, R., Natasha, M., \& Inge, Z. (2007). Effects of motor fatigue on human brain activity: An fMRI study. NeuroImage, 35, 1438-1449.

Yu, N., Hollnagel, C., Blickenstorfer, A., Kollias, S., \& Riener, R. (2008). fMRI-compatible robotic interfaces with fluidic actuation. In Proceedings of Robotics: Science and Systems IV, (pp. 199-205). Zurich, Switzerland: Eidgenössiche Technische Hochschule Zürich (ETHZ). 